\title{
Optical digital system for DOE computation
}

\author{
S.K. Misievich ${ }^{1,2}$, R.V. Skidanov ${ }^{1,2}$

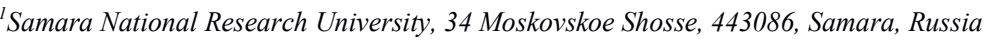 \\ ${ }^{2}$ Image Processing Systems Institute - Branch of the Federal Scientific Research Centre "Crystallography and Photonics" of Russian Academy of Sciences, 151 \\ Molodogvardeyskaya st., 443001, Samara, Russia
}

\begin{abstract}
This article considers algorithm implemented in an optoelectronic circuit. The main particularity of this algorithm is implementation in the working circuit of a part consisting of laser on the level of hardware, grey-level optical modulator of light and a diffraction pattern camera in the Fourier plane. This design allows using a laser with any initial distribution of intensity and adjusting a phase function of calculated DOE exactly for this distribution in order to decrease an error in forming a diffraction pattern of the output distribution.
\end{abstract}

Keywords: laser; light optical modulator; DOE; coding method; Fourier plane; Fourier transform

\section{Introduction}

For problems of synthesizing diffraction optical component (DOE) iterational (iterative) methods were developed and are used widely [1-11]. Their main advantage is that iterational algorithms prove to be more precise in comparison with other algorithms for DOE phase computation[6-10]. On the other hand, focusators computated with their help have irregular microrelief, which raises requirements for production technology of the components computated. Besides, the DOE computation using iterative algorithms requires significant expenses.

Disadvantage of algorithms executed with a computer is an implementation of intensity distribution approximation for a laser beam used as an illuminating beam for the DOE. This paper discusses an algorithm implemented in an optoelectronic circuit. The main particularity of this algorithm is implementation in the working circuit of a part consisting ofd with laser on the level of hardware, grey-level optical modulator of light and a diffraction pattern camera in the Fourier plane. Such design allows using a laser with any initial distribution of intensity and adjusting a phase function of calculated DOE exactly for this distribution in order to decrease an error in forming a diffraction pattern of the output distribution.

\section{Iterational algorithm}

The problem of image recovery in a lens focussing plane, set by its amplitude-phase distribution is reduced to problem of minimizing functionality of amplitude deviation in a recovered image from a set value [1-3]:

$$
\Phi=\left(|G(u, v)|^{2}-|F(u, v)|^{2}\right)
$$

where $|F(u, v)| и|G(u, v)|$ - is a set and calculated wave amplitude in the plane of spatial spectrum.

Let us take a coordinate descent algorithm as a basis for functionality minimizing algorithm (1). For this, solving onedimension problems of optimization shall be carried out with dichotomy method.

Let us use coefficients obtained with two-dimension unary re-expression [4] as coordinates for the coordinate descent:

$$
F(u, v)=\sum_{x=0}^{N-1} \sum_{y=0}^{M-1} F(x, y) A(u, v, x, y),
$$

where $A(u, v, x, y)$ - is the null-space for forward transformation;

$F(x, y)$ - size imaging matrix $N \times M$;

$F(u, v)$-transformed size imaging matrix $N_{1} \times M_{1}$.

In the process of transforming (2) an initial image is described by a set of coefficients, quantity of which is significantly lower than dimensions of the initial data, which in its turn facilitates computational speed.

Recover y of an initial image is carried out by means of inverse transformation:

$$
F(x, y)=\sum_{u=0}^{N_{1}-1} \sum_{v=0}^{M_{1}-1} F(u, v) B(u, v, x, y) \text {, }
$$

where $B(u, v, x, y)$ - is the null-space for the forward transformation;

One of possible forward and inverse transformations representations for images by size $N \times N$ may have form of forward and inverse Fourier transforms:

Let us compile algorithm scheme:

$$
\begin{aligned}
& F(u, v)=\frac{1}{N} \sum_{j=0}^{N-1} \sum_{k=0}^{N-1} F(j, k) \exp \left\{\frac{-2 \pi i}{N}(u j+v k)\right\}, \\
& F(j, k)=\frac{1}{N} \sum_{u=0}^{N_{1}-1} \sum_{v=0}^{N_{1}-1} F(u, v) \exp \left\{\frac{2 \pi i}{N}(u j+v k)\right\} .
\end{aligned}
$$

1) computation of expansion factors for a given initial approximation of a recovered image with expansion formula (3);

2) finding interval of expansion factor modification $F\left(u_{0}, v_{0}\right)$;

3) carrying out of functionality minimizing with dichotomy method computated at previous stage of interval algorithm:

1. image recovery using known expansion coordinates with an inverse transform (4);

2. performing Fourier transform for obtaining distribution in lens focussing plane; 
3. functionality recalculating (1) in relation to the distribution deduced;

4. calculation of functionality Euclidean norm;

5. finding interval of expansion factor modification;

6. checking exit condition from dichotomy method;

4) checking exit condition from algorithm. Exit is effected in case we achieved functionality minimum with a set definiteness or we reset all the expansion factors.

At realization of this algorithm in an optoelectronic circuit, Fourier transform performance, which make a significant part of computations is transferred to hardware component [5]. Realization of these calculations is carried out by means of spatial light modulator, which modifies amplitude of illuminating beam. After this, the light passes through a collecting lens, forming a distribution in focussing plane, which is recorded by the camera and serves as a basis for functionality recalculation (1).

\section{Experimental research}

Let us conduct a simulation experiment for a DOE phase function optimization using a worked out iterational algorithm.

Let us use a result received with the iterational algorithm as initial approximation of a phase. We conduct results of numerical experiments for images that describe amplitude and phase distributions with dimensions of $256 \times 256$ pixels . For acceleration of the algorithm work we employ radially-symmetrical phase and amplitude distributions as investigated.

A ring given at figure 11 is used as a reference distribution.

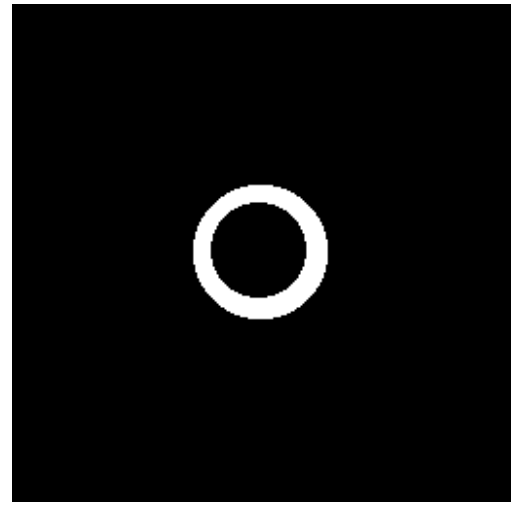

Fig. 1. Reference distribution of intensity.

As a method for inaccuracy estimation, a mean square deviation was used:

$$
\varepsilon=\frac{\sqrt{\frac{1}{s} \sum_{(x, y) \in R}[I(x, y)-\grave{I}(x, y)]^{2}}}{\frac{1}{s} \sum_{(x, y) \in R} \grave{I}(x, y)},
$$

where $I(x, y)$ - is the distribution of the intensity formed;

$\grave{I}(x, y)$ - reference distribution of intensity;

$R$ - area of inaccuracy estimation;

$S$ - space of the area $R$.

For the calculated initial approximation, inaccuracy was 0.81 . After implementation of iterational algorithm, the inaccuracy reduced to 0.48 . Results of the algorithm performance a re given in figure 2 .
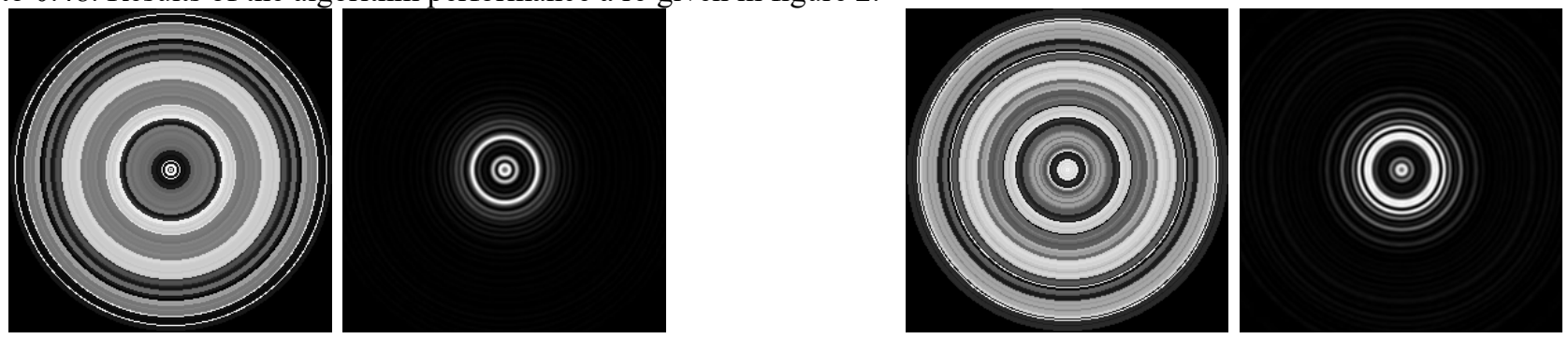

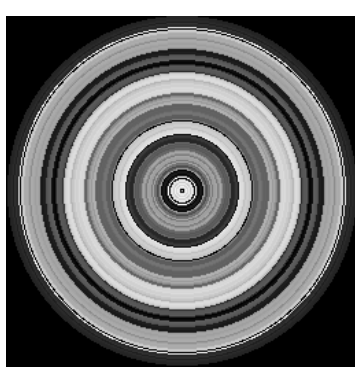

a)

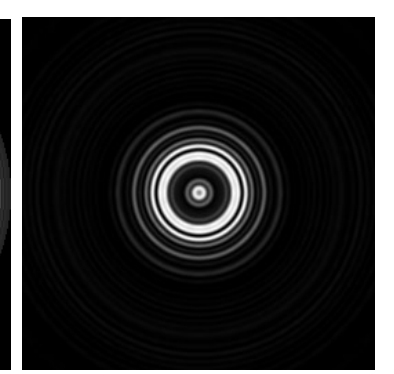

b)

Fig. 2. Phase (a) and it's corresponding intensity (b) for consecutive iterations of the algorithm with inaccuracy level 0,$67 ; 0,54 ; 0,48$ correspondingly.

Carrying out simulation implementing various initial approximations, we obtained results given in figures 3 and 4. 


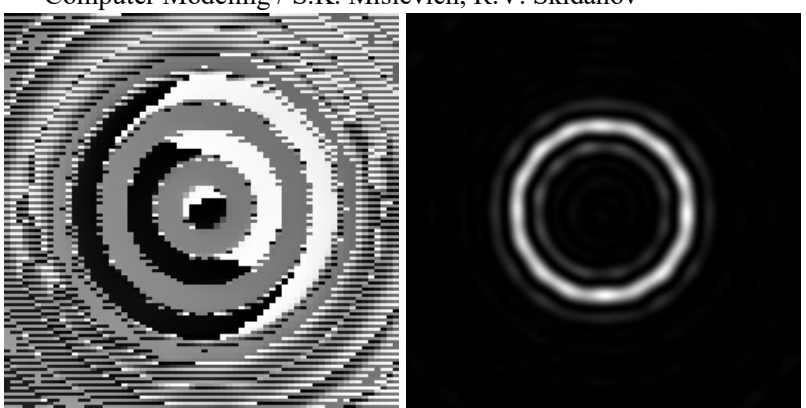

a)
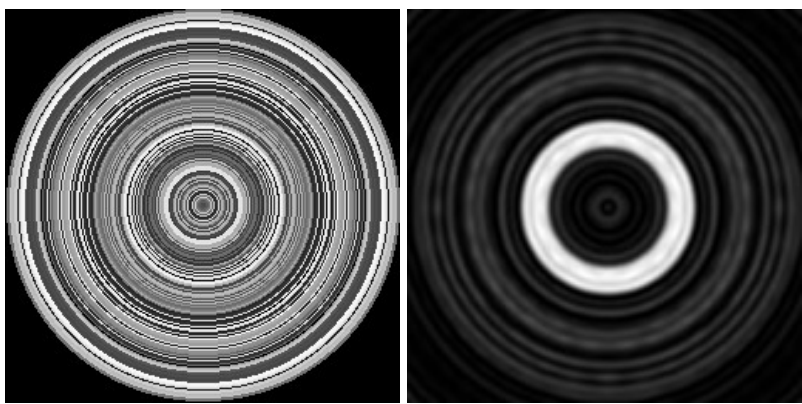

b)

Fig. 3. DOE phase and intensity obtained from Fourier plane for the initial approximation, which is computated using method of local phase jump (a) and at exit of the ALGORITHM PERFORMACE (B).
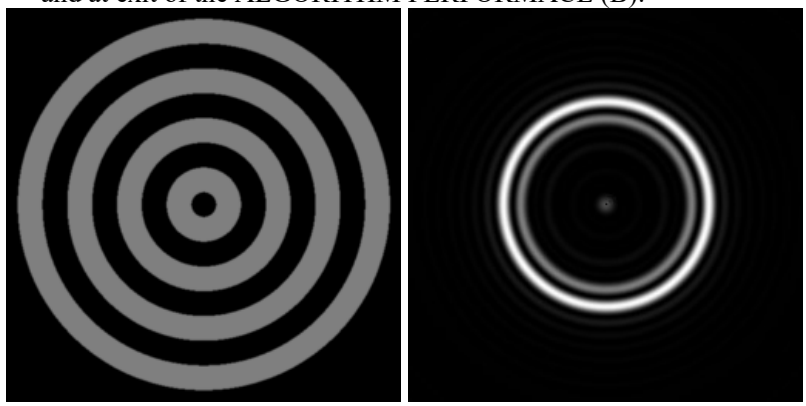

a)
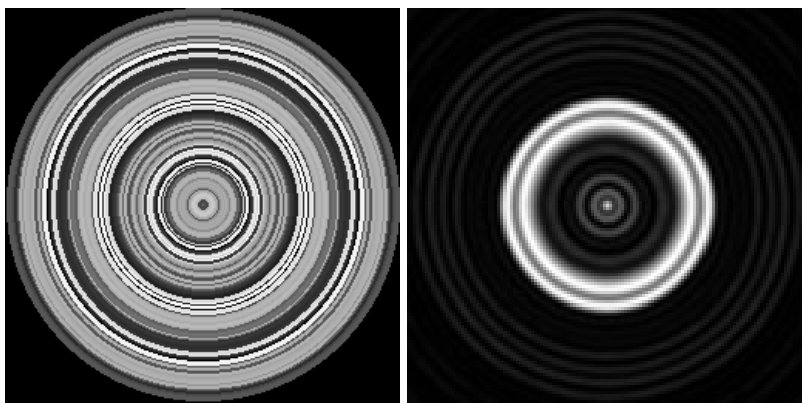

b)

Fig. 4. DOE phase and intensity obtained from Fourier plane for the initial approximation, which is an axicon (a) and at the exit of the algorithm's performance (b).

Fig. 3 and 4 show relative recovery inaccuracies, $25 \%$ and $32 \%$ correspondingly. Collation of the results obtained proves decrease in the algorithm's convergence speed when initial approximation increases.

To conduct an experiment, an optical design was built (see figure 15), with a grey-level optical modulator of light, model SLM PLUTO Phase Only [10,11,14].

At the exit of the laser a Fourier correlator is installed consisting of two lens (CL1, CL2) with different focal distance for beam blooming to a size that is capable of covering work panel of the modulator. Immediately before the modulator, the diaphragm is installed, which is necessary for varying diameter of the illuminating beam and for illuminating a certain area of the modulator. The laser beam falling onto the work panel of the modulator attached to the PC, changes its intensity and returns on the same trajectory. Reaching the splitter (SP), the beam divides in two parts, one of which as reflected by the mirror (M) and passing through a convex lens (CL3) forms some distribution in the Fourier plane. The distribution obtained is recorded by the camera attached to the PC displaying a formed diffraction pattern. The optical scheme was also equipped with different darkening optical filters (OF). 


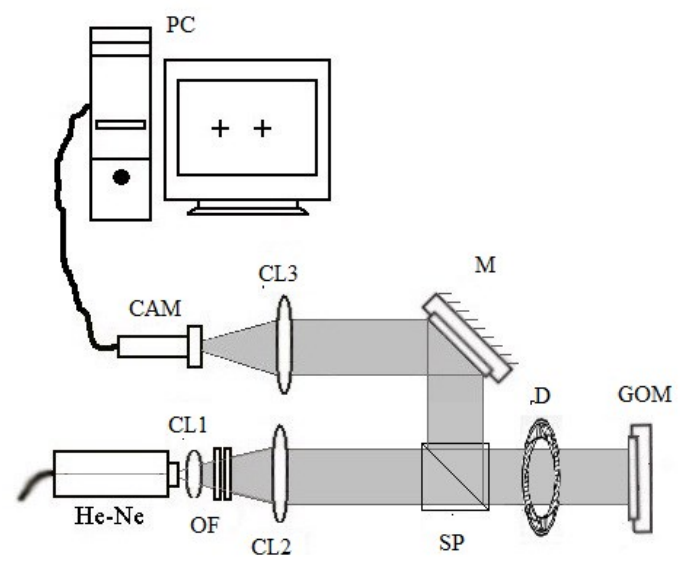

He-Ne - helium-neon solid-state laser, OF - optical filters, CL1, CL2, CL3 - convex lens, SP - splitter, D - diaphragm, GOM - grey-level spatial optical modulator CRL OPTO, CAM - camera VSTT-252, M - rotating mirror, PC - personal computer

Fig. 5. Optical scheme used in the experiment.

Figure 6 shows a photography of the optoelectronic system in action.

Implementation of such scheme in an algorithm for computating DOE phase function is that the phase function corrected at each algorithm's iteration is brought off to the modulator, which functions as an actual DOE, then the camera records the diffraction pattern in the lens focussing plane. Further, the distribution obtained is processed by the computer. Discrepancy error between the reference and the obtained distribution is calculated and an operation of the phase correction occurs.

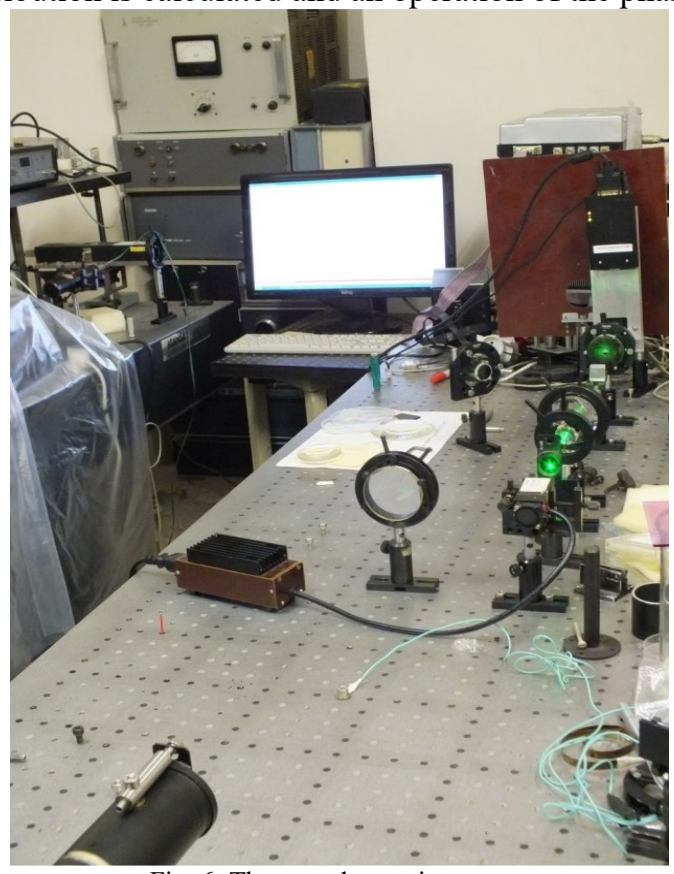

Fig. 6. The optoelectronic system.

As an initial approximation of the intensity formed we shall employ DOE shown on figure 3a. After its forming on the optical scheme, the distribution shown on fig. 7 was received.

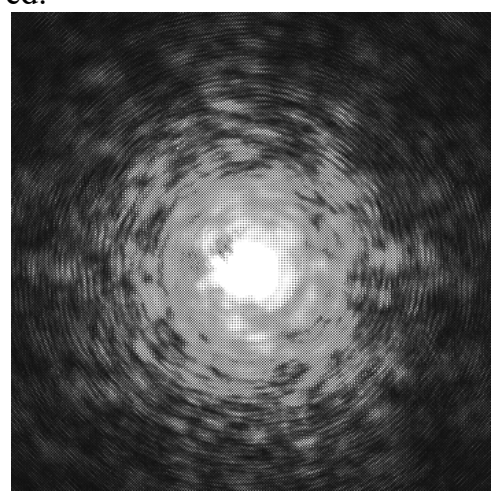

Fig. 7. The reference distribution of intensity.

Such distribution possesses a significant peak related to re-reflection effect, which occurs in the modulator. Further, fig. 8 shows the result of a programme deduction from the distribution, which describes this peak. 


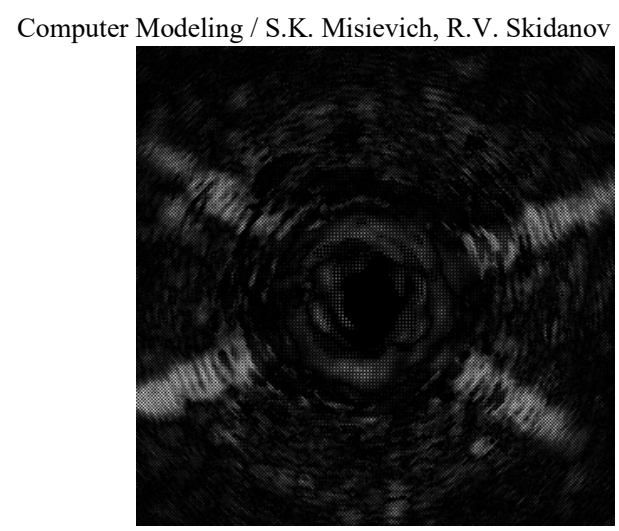

Fig. 8. The difference of intensity.

The calculated deviation of this intensity distribution from the reference one makes approx. 95\%.

The result of the algorithm's work, which is implementedd in the optoelectronic circuit is given in fig. 9. The distribution corresponding it, as obtained by means of diminution from the central peak is given in fig. 10. The DOE phase function computated has the form represente din fig. 11.

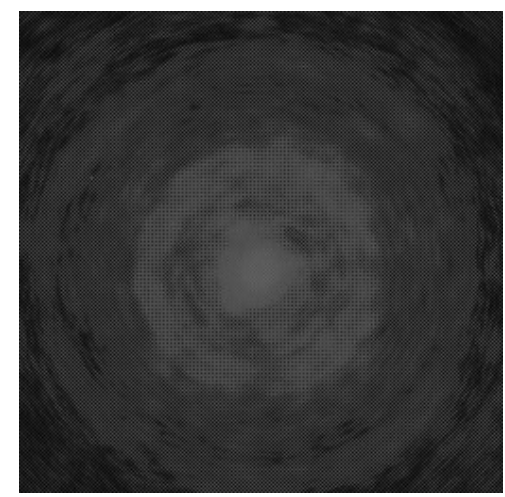

Fig. 9. The resultant distribution of intensity.

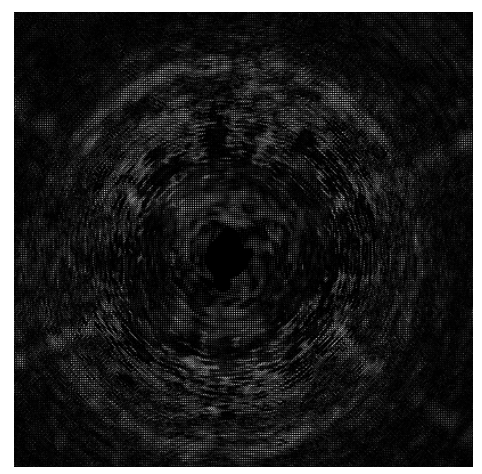

Fig. 10. The result of the programme deduction in the central peak.

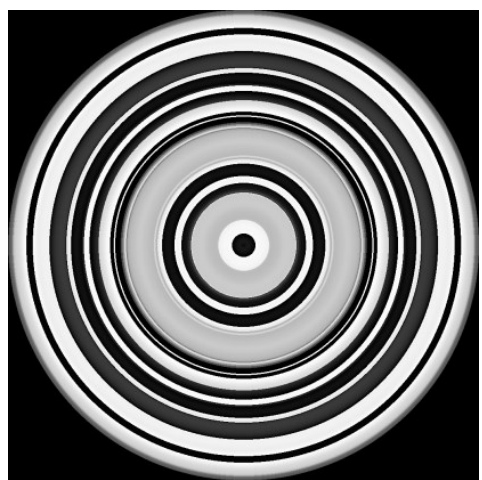

Fig. 11. The calculated DOE phase.

The distribution obtain as a result of the algorithm performance has a more a more distinct ringed structure, which corresponds more to the set reference distribution. The calculated deviation of this image from the reference one makes $83 \%$.

An essential disadvantage of such computation method for the DOE phase function is a low speed of the algorithm's work, which correlated to output of a frequently alternating phase distribution onto the modulator. 


\section{Conclusion}

In work process, an algorithm for calculating the DOE phase function was elaborated, which is used for forming a radiallysymmetrical distribution in the Fourier plane of the convex lens. Such algorithm was implementedd on the computer for conducting a work simulation and convergence research, then transposed to the optoelectronic circuit.

The results of conducting a live experiment showed possibility of implementing this algorithm for calculating the diffraction optical components. The main advantage of the algorithm is its universality in relation to the intensity distribution of the illuminating beam. When calculating the DOE phase function, there is no need for taking into account laser intensity distribution, as it is counted on the hardware level, as a result of the algorithm's work the phase function is corrected exactly for the given distribution. Although, a low speed of the algorithm's work in the optoelectronic circuit hinders its implementation.

Such algorithm may be used in cases, when it is necessary to calculate a component that gives a more distinct image at the output, using a laser with a specific intensity distribution.

\section{Acknowledgements}

The work was funded by the Russian Federation Ministry of Education and Science of state-assigned task No. $3.3025 .2017 / 8.9$

\section{References}

[1]. Lanina EP. Organization of ECM and systems. Site of Irkutsk State Technical University, 2004. URL: http://paralichka85.px6.ru/11future/glava11 1.htm (date of reference: 09.01.2013).

[2]. Kotlyar VV, Khonina SN, Melekhin AS, Soifer VA. Coding of diffraction optical components using method of phase jump. Computer Optics 1999; 19(9): 54-64.

[3]. Soifer VA, Doskolovich LL, Golovashkin DL, Kazanskiy NL, Kharitonov SI, Khonina SN, Kotlyar VV, Pavelyev VS, Skidanov RV, Solovyev VS, Uspleniev GV, Volkov AV. Methods for computer design of diffractive optical elements. New York: John Wiley \& Sons, Inc., 2002.

[4]. Pratt W. Digital processing of images. Moscow: Mir Publishing house, 1982; 1: 312 p.

[5]. Fast spatial light modulators speed optical-computing applications. Vision Systems Design, 1997. URL: http://www.visionsystems.com/articles/print/volume-2/issue-8/applications/spotlight/fast-spatial-light-modulators-speed-optical-computing-applications.html (date of reference: 23.12.2012).

[6]. Kazanskiy NL, Kotlyar VV, Soifer VA. Computer-aided design of diffractive optical elements. Optical Engineering 1994; 33: 3156-3166. DOI: $10.1117 / 12.178898$.

[7]. Doskolovich LL, Golub MA, Kazanskiy NL, Khramov AG, Pavelyev VS, Seraphimovich PG, Soifer VA, Volotovskiy SG. Software on diffractive optics and computer generated holograms. Proceedings of SPIE 1995; 2363: 278-284.

[8]. Golovashkin DL, Kasanskiy NL. Solving diffractive optics problem using graphics processing units. Optical Memory and Neural Networks (Information Optics) 2011; 20: 85-89. DOI: 10.1134/S1063776110120095.

[9]. Kharitonov SI, Doskolovich LL, Kazanskiy NL. Solving the inverse problem of focusing laser radiation in a plane region using geometrical optics. Computer Optics 2016; 40(4): 439-450. DOI: 10.18287/2412-6179-2016-40-4-439-450.

[10]. Kazanskiy NL. Research and education center of diffractive optics. Proceedings of SPIE 2012; 8410: 84100R. DOI: 10.1117/12.923233.

[11]. Kovalev AA, Kotlyar VV, Porfirev AP. Generation of half-pearcey laser beams by a spatial light modulator. Computer Optics $2014 ; 38$ (4) 658-662.

[12]. Method of descent by coordinates. Multi-dimensional methods of optimization. URL: http://school-sector.relarn.ru/dckt/projects/optim/pocspusc.htm (date of reference: 15.12 .2012 ).

[13]. Dichotomy method. Encyclopedias and dictionaries. URL: http://dic.academic.ru/dic.nsf/ruwiki/1034684 (date of reference: 17.12.2012).

[14]. PLUTO: High-Resolution LCOS Phase Only Spatial Light Modulators. HOLOEYE Pioneers in Photonic Tecnology, 1997. URL: http://www.holoeye.com/spatial_light_modulators_pluto.html (date of reference: 15.01.2012). 